\title{
Microbiology Susceptibility Elapsed Time
}

National Cancer Institute

\section{Source}

National Cancer Institute. Microbiology Susceptibility Elapsed Time. NCI Thesaurus. Code C87931.

The interval between two microbiology susceptibility reference time points. 